International Journal of Pure and Applied Mathematics

Volume 114 No. $4 \quad 2017,857-866$

ISSN: 1311-8080 (printed version); ISSN: 1314-3395 (on-line version)

url: http://www.ijpam.eu

doi: 10.12732/ijpam.v114i4.15

ijpam.eu

\title{
CHARACTERIZATION THEOREM FOR AN INDEFINITE ALMOST COMPLEX MANIFOLD WITH NORDEN METRIC
}

\author{
Anu Devgan ${ }^{1 \S}$, Rakesh Kumar Nagaich ${ }^{2}$ \\ ${ }^{1,2}$ Department of Mathematics \\ Punjabi University \\ Patiala, 147002, INDIA
}

\begin{abstract}
We derive the expression for a Riemannian curvature tensor for a Kaehler manifold with Norden metric. Then we obtain a characterization theorem for an indefinite almost complex manifold with Norden metric to be a space of constant holomorphic sectional curvature.
\end{abstract}

AMS Subject Classification: $53 \mathrm{C} 15,53 \mathrm{C} 50,53 \mathrm{C} 56$

Key Words: almost complex manifold, norden metric, holomorphic sectional curvature

\section{Introduction}

The theory of submanifolds of an almost complex manifold is considered as an interesting topic in differential geometry for the behaviour of the tangent bundle of the submanifold with respect to the action of the almost complex structure $J$. The basic difference between the geometry of an almost complex manifold with Hermitian metric and an almost complex manifold with Norden metric is that the action of the almost complex structure $J$ on the tangent space is isometry with respect to the Hermitian metric and the action of almost complex structure $J$ is anti-isometry with respect to the Norden metric. Almost complex manifolds with Norden metric were first studied by Norden [9]. Ganchev et al.

Received: February 23, 2017

Revised: $\quad$ May 20, 2017

Published: June 7, 2017

$\S$ Correspondence author (c) 2017 Academic Publications, Ltd. url: www.acadpubl.eu 
[3], obtained a classification of almost complex manifold with Norden metric with respect to the covariant derivative of the almost complex structure. In 1993, Ganchev et al. [4] introduced the geometry of almost contact B-metric (Norden metric) manifolds and obtained a classification of the almost contact manifolds with B-metric.

In [10], Tano provided an algebraic characterization for an almost Hermitian manifold to reduce to a space of constant holomorphic sectional curvature, which he later extended for the Sasakian manifolds as well. Nagaich [8] generalized the characterization of Tano for almost Hermitian manifolds to indefinite almost Hermitian manifolds and further Kumar et al. [6], generalized the characterization of Tano for Sasakian manifolds to indefinite Sasakian manifolds. Since there are significant applications of almost complex manifolds with Norden metric in Theoretical Physics and Spinor theory [1].

Therefore all these factors motivated us to study on this subject matter. In this paper, we obtain a characterization theorem for an indefinite almost complex manifold with Norden metric to be a space of constant holomorphic sectional curvature.

\section{Almost Complex Manifolds with Norden Metric}

Let $(M, J, g)$ be a 2 n-dimensional almost complex manifold with Norden metric [3], that is, $J$ is an almost complex structure and $g$ is a metric on $M$ such that

$$
J^{2} X=-X, \quad g(J X, J Y)=-g(X, Y),
$$

for all differentiable vector fields $\mathrm{X}, \mathrm{Y}$ on $M$. The tensor field $\tilde{g}$ defined by

$$
\tilde{g}(X, Y)=g(J X, Y)
$$

is a Norden metric on $M$, too. Both the metrics $g$ and $\tilde{g}$ are necessarily of signature $(n, n)$. The metric $\tilde{g}$ is called an associated metric of $M$.

An almost complex manifold with Norden metric $(M, g, J)$ is said to be a Kaehler manifold with Norden metric if $\nabla J=0$ with respect to the Levi-Civita connection $\nabla$ of $g$. Let $R$ be the curvature tensor of $\nabla$ and given by

$$
R(X, Y) Z=\nabla_{X} \nabla_{Y} Z-\nabla_{Y} \nabla_{X} Z-\nabla_{[X, Y]} Z,
$$

for all differentiable vector fields $\mathrm{X}, \mathrm{Y}$ and $\mathrm{Z}$ on $M$. The curvature tensor $R$ of the type $(0,4)$ is defined by $R(X, Y, Z, U)=g(R(X, Y) Z, U)$ for all $X, Y, Z, U \in$ $T_{p} M, p \in M$, and has the property

$$
R(X, Y, Z, U)=-R(X, Y, J Z, J U) .
$$


This implies that the curvature tensor $\tilde{R}$ of $\tilde{\nabla}$ is defined by

$$
\tilde{R}(X, Y, Z, U)=R(X, Y, Z, J U)
$$

and has the property

$$
\tilde{R}(X, Y, Z, U)=-\tilde{R}(X, Y, U, Z) .
$$

Hence for the curvature tensor $R$, we have

$$
R(X, Y, Z, J U)=R(X, Y, J Z, U) .
$$

We categorize a vector field $X$ in three types with respect to metric $g$ as

- spacelike if $g(X, X)>0$.

- timelike if $g(X, X)<0$.

- null (lightlike) if $g(X, X)=0, X \neq 0$.

If for the vector fields $X, Y$ where $(X \neq 0)$ and $g(X, Y)=0$ for every $Y$, then $g$ is called a degenerate metric and the plane $\operatorname{span}\{X, Y\}$ is called a degenerate plane if and only if

$$
g(X, X) g(Y, Y)-g(X, Y)^{2}=0
$$

The sectional curvature for a non-degenerate plane $\operatorname{span}\{X, Y\}$ is defined as

$$
K(X, Y)=\frac{R(X, Y, X, Y)}{g(X, X) g(Y, Y)-g(X, Y)^{2}} .
$$

The value of holomorphic sectional curvature $H(X)$, for a unit tangent vector $X$ is the sectional curvature $K(X, J X)$. If $H(X)$ is always constant $(=\mathrm{c}(\mathrm{p}))$ with respect to any unit vector $X \in T_{p}(M)$ and at a point $p \in M$, then $\mathrm{M}$ is said to be of constant holomorphic sectional curvature or a complex space form and denoted by $M(c(p))$. Since the holomorphic sectional curvatures are of fundamental importance, so our first aim is to find the expression of a Riemannian curvature tensor for a Kaehler manifold $M$ with Norden metric having constant holomorphic sectional curvature. We have the following Lemma from [5], which is useful in finding the required expression of Riemannian curvature.

Lemma 1. Let $M$ be a nearly Kaehler manifold and the quadratic holomorphic curvature form $Q$ defined by $Q(X)=K(X, J X, X, J X)$. Then for any vectors $X, Y \in T_{m} M$, the sectional curvature is given by

$$
R(X, Y, X, Y)=-\frac{1}{32}[3 Q(X+J Y)+3 Q(X-J Y)-Q(X+Y)
$$




$$
-Q(X-Y)-4 Q(X)-4 Q(Y)]-\frac{3}{4}\left\|\left(\nabla_{X} J\right) Y\right\|^{2},
$$

where $R(X, Y, X, Y)-R(X, Y, J X, J Y)=\left\|\left(\nabla_{X} J\right) Y\right\|^{2}$.

Since for a Kaehler manifold, $R(X, Y, X, Y)=R(X, Y, J X, J Y)$, therefore for a Kaehler manifold (3) becomes

$$
\begin{aligned}
R(X, Y, X, Y)= & -\frac{1}{32}[3 Q(X+J Y)+3 Q(X-J Y)-Q(X+Y) \\
& -Q(X-Y)-4 Q(X)-4 Q(Y)],
\end{aligned}
$$

for any vectors $X, Y \in T_{m} M$.

Theorem 2. Let $(M, g, J)$ be a Kaehler manifold with Norden metric having constant holomorphic sectional curvature $c(p)$ at every point $p \in M$, then the Riemannian curvature tensor of $M$ is of the form

$$
\begin{aligned}
R(X, Y, Z, W)=\frac{c(p)}{4}[ & g(X, Z) g(Y, W)-g(X, W) g(Y, Z) \\
& -g(X, J W) g(Z, J Y)+g(X, J Z) g(Y, J W)]
\end{aligned}
$$

for any vectors $X, Y, Z, W \in T_{p}(M)$.

Proof. Let $X, Y \in T_{p}(M)$ then

$$
\begin{aligned}
\|X \pm J Y\|^{4}= & g(X \pm J Y, X \pm J Y)^{2} \\
= & (g(X, X)-g(Y, Y))^{2} \pm 4(g(X, X)-g(Y, Y)) g(X, J Y) \\
& +4 g(X, J Y)^{2},
\end{aligned}
$$

and

$$
\begin{aligned}
\|X \pm Y\|^{4}= & g(X \pm Y, X \pm Y)^{2} \\
= & (g(X, X)+g(Y, Y))^{2} \pm 4(g(X, X)+g(Y, Y)) g(X, Y) \\
& +4 g(X, Y)^{2} .
\end{aligned}
$$

If using (2), we put $Q(X)=R(X, J X, X, J X)=-H(X)\|X\|^{4}$ then we have

$$
\begin{aligned}
Q(X+J Y)= & -H(X+J Y)\|X+J Y\|^{4} \\
= & -H(X+J Y)\left[(g(X, X)-g(Y, Y))^{2}\right. \\
& \left. \pm 4(g(X, X)-g(Y, Y)) g(X, J Y)+4 g(X, J Y)^{2}\right] .
\end{aligned}
$$


Similarly, calculating $Q(X-J Y), Q(X+Y)$ and $Q(X-Y)$ and substituting these results in (4), we have

$$
\begin{aligned}
R(X, Y, X, Y)= & -\frac{1}{32}\left[3 H ( X + J Y ) \left\{(g(X, X)-g(Y, Y))^{2}\right.\right. \\
& \left.+4(g(X, X)-g(Y, Y)) g(X, J Y)+4 g(X, J Y)^{2}\right\} \\
& +3 H(X-J Y)\left\{(g(X, X)-g(Y, Y))^{2}\right. \\
& \left.-4(g(X, X)-g(Y, Y)) g(X, J Y)+4 g(X, J Y)^{2}\right\} \\
& -H(X+Y)\{g(X, X)+g(Y, Y))^{2} \\
& \left.+4(g(X, X)+g(Y, Y)) g(X, Y)+4 g(X, Y)^{2}\right\} \\
& -H(X-Y)\left\{(g(X, X)+g(Y, Y))^{2}\right. \\
& \left.-4(g(X, X)+g(Y, Y)) g(X, Y)+4 g(X, Y)^{2}\right\} \\
& \left.-4 H(X) g(X, X)^{2}-4 H(Y) g(Y, Y)^{2}\right] .
\end{aligned}
$$

Since $M$ is of constant holomorphic sectional curvature $c(p)$ therefore from (6), we obtain

$$
R(X, Y, X, Y)=\frac{c(p)}{4}\left[g(X, Y)^{2}+2 g(X, X) g(Y, Y)-3 g(X, J Y)^{2}\right] .
$$

Replacing $Y$ by $Y+W$ in (7), we get

$$
\begin{aligned}
R(X, Y, X, W)= & \frac{c(p)}{4}[g(X, Y) g(X, W)+2 g(X, X) g(Y, W) \\
& -3 g(X, J Y)(X, J W)]
\end{aligned}
$$

and replacing $X$ by $X+Z$, we have

$$
\begin{aligned}
& R(X, Y, Z, W)+R(Z, Y, X, W)=\frac{c(p)}{4}[g(X, Y) g(Z, W)+g(Z, Y) g(X, W) \\
& +4 g(X, Z) g(Y, W)-3 g(X, J Y)(Z, J W)-3 g(Z, J Y)(X, J W)] .
\end{aligned}
$$

Interchanging $X$ and $Y$ in (9) and subtracting the resulting equation from (9), we obtain

$$
\begin{aligned}
R & (X, Y, Z, W)+R(Z, Y, X, W)-R(Y, X, Z, W)-R(Z, X, Y, W) \\
= & \frac{c(m)}{4}[g(X, Y) g(Z, W)+g(Z, Y) g(X, W)+4 g(X, Z) g(Y, W) \\
& -3 g(X, J Y)(Z, J W)-3 g(Z, J Y)(X, J W)-g(Y, X) g(Z, W) \\
& -g(Z, X) g(Y, W)-4 g(Y, Z) g(X, W)+3 g(Y, J X)(Z, J W) \\
& +3 g(Z, J X)(Y, J W)] .
\end{aligned}
$$

Hence using the first Bianchi's identity, the expression follows. 


\section{Constancy of Holopmorphic Sectional Curvature}

Tano [10] provided an algebraic characterization for an almost Hermitian manifold to reduce to a space of constant holomorphic sectional curvature as

Theorem 3. Let dimension $(2 n \geq 4)$, assume that almost Hermitian manifold $\left(M^{2 n}, g, J\right)$ satisfies

$$
R(J X, J Y, J Z, J X)=R(X, Y, Z, X),
$$

for every tangent vectors $X, Y$ and $Z$. Then $\left(M^{2 n}, g, J\right)$ is of constant holomorphic sectional curvature at $x$, if and only if, $R(X, J X) X$ is proportional to $J X$, for every tangent vector $X$ at $p$ in $M$.

Further Nagaich [8] generalized the above characterization for an indefinite almost Hermitian manifold as:

Theorem 4. Let $\left(M^{2 n}, g, J\right)(2 n \geq 4)$ be an indefinite almost Hermitian manifold satisfying (11). Then $M$ is of constant holomorphic sectional curvature at $p$, if and only if, $R(X, J X) X$ is proportional to $J X$, for every tangent vector $X$ at $p$.

Tano [10] also extended the Theorem (3) for the Sasakian manifolds as well and then Kumar et al.[6] generalized the same characterization for indefinite Sasakian manifolds as:

Theorem 5. Let $\left(M^{2 n+1}, \phi, \eta, \xi, g\right)(2 n \geq 4)$ be an indefinite Sasakian manifold. Then $\left(M^{2 n+1}\right)$ is of constant $\phi$-sectional curvature if and only if $R(X, \phi X) X$ is proportional to $\phi X$ for every tangent vector field $X$ such that $g(X, \xi)=0$, where $\xi$ is a characteristic vector field of $M$.

Theorem 6. Let $\left(M^{2 n}, g, J\right)(2 n \geq 4)$ be an indefinite almost complex manifold with Norden mertic satisfying (11). Then $\left(M^{2 n}, g, J\right)$ is of constant holomorphic sectional curvature at $p$ if and only if

$$
R(X, J X) X \quad \text { is proportional to } \alpha X+\beta J X,
$$

where $\alpha$ and $\beta$ are the functions of holomorphic sectional curvature $H(X)$, for every tangent vector $X$ at $p \in M$.

Proof. Let $M$ be an indefinite almost complex manifold with Norden metric such that $M$ is of constant holomorphic sectional curvature at $p$. Let $X$ be a unit vector such that $g(X, J X)=k(\neq 0)$ then using (2), we have

$$
R(X, J X) X=-H(X) k X+H(X) J X .
$$


Since $M$ is of constant holomorphic sectional curvature at $p$ hence the necessity of the assertion follows from (12). Conversely, we shall consider the following two cases:

$$
\begin{gathered}
\text { (i) } g(X, X)=g(Y, Y) \text { and } \\
\text { (ii) } g(X, X)=-g(Y, Y) .
\end{gathered}
$$

For first case, let $\{X, Y\}$ be a pair of orthonormal vectors in $M$ such that $g(X, X)=-g(J X, J X)=1, g(Y, Y)=-g(J Y, J Y)=1, g(X, J X)=g(Y, J Y)=$ $k(\neq 0)$ constant and $g(X, J Y)=g(J X, Y)=0$. Now, define $X^{*}$ and $Z^{*}$ by $X^{*}=\cos \theta X+\sin \theta Y$ and $Z^{*}=-\sin \theta X+\cos \theta Y$, then $\left\{X^{*}, Z^{*}\right\}$ form a pair of orthonormal vectors in $M$ therefore,

$$
R\left(X^{*}, J X^{*}\right) X^{*} \text { is proportional to } \alpha X^{*}+\beta J X^{*} \text {. }
$$

Taking inner product with $J Z^{*}$, we get

$$
R\left(X^{*}, J X^{*}, X^{*}, J Z^{*}\right)=0 .
$$

Using the linear properties of Riemannian curvature tensor $R$, we obtain

$$
\begin{aligned}
& \cos \theta \sin \theta\left\{-\cos ^{2} \theta R(X, J X, X, J X)+\sin ^{2} \theta R(Y, J Y, Y, J Y)\right. \\
& \left.+\left(\cos ^{2} \theta-\sin ^{2} \theta\right) R(Y, J Y, X, J X)\right\}=0 .
\end{aligned}
$$

Taking $\theta=\frac{\pi}{4}$, we get

$$
H(X)=H(Y) .
$$

If $\{U, V\}$ is an holomorphic section then $J U=p U+q V$, for any scalars $P$ and $q$. Then $\{U, J U\}=\{U, p U+q V\}=\{U, V\}$ and similarly $\{V, J V\}=\{U, V\}$. Therefore $\{U, J U\}=\{V, J V\}$ and hence $H(U)=H(V)$. If $\{U, V\}$ is not an holomorphic section then there must exist unit vectors $X \in\{U, J U\}^{\perp}$ and $Y \in\{V, J V\}^{\perp}$, which determine an holomorphic section $\{X, Y\}$ and thus we get

$$
H(U)=H(X)=H(Y)=H(V) .
$$

This gives that any holomorphic section has same holomorphic sectional curvature.

Now assume that the dimension of $M=4$ such that then using the properties of curvature tensor $R$, we have the following relations

$$
\begin{gathered}
R(X, J X) X=H(X)\{-k X+J X\} \\
R(X, J X) Y=\frac{1}{1+k^{2}}\{R(X, J X, Y, J Y)(k Y-J Y)\}
\end{gathered}
$$




$$
\begin{aligned}
& R(X, J Y) X= \frac{1}{1+k^{2}}\{R(X, J Y, X, Y)(Y+k J Y)+R(X, J Y, X, J Y)(k Y-J Y)\} \\
& R(Y, J X) X= \frac{1}{1+k^{2}}\{R(Y, J X, X, Y)(Y+k J Y)+R(Y, J X, X, J Y)(k Y-J Y)\} \\
& R(X, J Y) Y= \frac{1}{1+k^{2}}\{R(X, J Y, Y, X)(X+k J X)+R(X, J Y, Y, J X)(k X-J X)\} \\
& R(Y, J X) Y= \frac{1}{1+k^{2}}\{R(Y, J X, Y, X)(X+k J X)+R(Y, J X, Y, J X)(k X-J X)\} \\
& R(Y, J Y) X=\frac{1}{1+k^{2}}\{R(Y, J Y, X, J X)(k X-J X)\} \\
& R(Y, J Y) Y=H(Y)\{-k Y+J Y\} .
\end{aligned}
$$

Now define $X^{*}=a X+b Y$ with $a^{2}+b^{2}=1$, then using the above relations, we have

$$
R\left(X^{*}, J X^{*}\right) X^{*}=C_{1} X+C_{2} Y+C_{3} J X+C_{4} J Y
$$

where

$$
C_{3}=a^{3} H(X)-\frac{a b^{2}}{\left(1+k^{2}\right)} C_{5}, \quad C_{4}=b^{3} H(X)-\frac{a^{2} b}{\left(1+k^{2}\right)} C_{5},
$$

and $C_{5}=R(X, J Y, Y, J X)+R(Y, J X, Y, J X)+R(Y, J X, X, J X)$. On the other hand, using (14), we have

$$
R\left(X^{*}, J X^{*}\right) X^{*}=H\left(X^{*}\right)\left\{-k X^{*}+J X^{*}\right\}=H\left(X^{*}\right)\{k a X+k b Y-a J X-b J Y\} .
$$

Comparing (15) and (16), we obtain

$$
a^{2} H(X)-\frac{b^{2}}{\left(1+k^{2}\right)} C_{5}=H\left(X^{*}\right), \quad b^{2} H(X)-\frac{a^{2}}{\left(1+k^{2}\right)} C_{5}=H\left(X^{*}\right),
$$

from these equations, we obtain $C_{5}=-\left(1+k^{2}\right) H(X)$ and consequently $H\left(X^{*}\right)=$ $\left(a^{2}+b^{2}\right) H(X)=H(X)$. Similarly, we can prove $H\left(Y^{*}\right)=H(Y)$. Thus $M$ is of constant holomorphic sectional curvature at $p$.

For the second case, let $\{X, Y\}$ be a pair of orthonormal vectors in $M$ such that $g(X, X)=-g(J X, J X)=1, g(Y, Y)=-g(J Y, J Y)=-1, g(X, J X)=$ $-g(Y, J Y)=k(\neq 0)$ and $g(X, J Y)=g(J X, Y)=0$. Define $X^{\prime}$ and $Z^{\prime}$ by $X^{\prime}=\cosh \theta X+\sinh \theta Y$ and $Z^{\prime}=-\sinh \theta J X+\cosh \theta J Y$ then $X^{\prime}, Z^{\prime}$ form an orthonormal pair of vectors in $M$ therefore

$$
R\left(X^{\prime}, J X^{\prime}\right) X^{\prime} \quad \text { is proportional to } \alpha X^{\prime}+\beta J X^{\prime} .
$$


Taking inner product with $Z^{\prime}$, we get $R\left(X^{\prime}, J X^{\prime}, X^{\prime}, Z^{\prime}\right)=0$, and using the linear properties of curvature tensor, we obtain

$$
\begin{aligned}
& \cosh \theta \sinh \theta\left\{\cos ^{2} h \theta H(X)-\sin ^{2} h \theta H(Y)\right. \\
& \left.-\left(\cos ^{2} h \theta-\sin ^{2} h \theta\right) R(X, J X, Y, J Y)\right\}=0 .
\end{aligned}
$$

Taking $\theta=\frac{i \pi}{4}$, we get $H(X)=H(Y)$. Now assume that the dimension of $M=4$ then using the curvature properties of curvature tensor $R$, we have the following relations

$$
\begin{gathered}
R(X, J X) X=-H(X)\{k X-J X\} \\
R(X, J X) Y=\frac{1}{1+k^{2}}\{R(X, J X, Y, J Y)(-k Y+J Y)\} \\
R(X, J Y) X=\frac{1}{1+k^{2}}\{R(X, J Y, X, Y)(-Y-k J Y)+R(X, J Y, X, J Y)(-k Y+J Y)\} \\
R(Y, J X) X=\frac{1}{1+k^{2}}\{R(Y, J X, X, Y)(-Y-k J Y)+R(Y, J X, X, J Y)(-k Y+J Y)\} \\
R(X, J Y) Y=\frac{1}{1+k^{2}}\{R(X, J Y, Y, X)(X+k J X)+R(X, J Y, Y, J X)(k X-J X)\} \\
R(Y, J X) Y=\frac{1}{1+k^{2}}\{R(Y, J X, Y, X)(X+k J X)+R(Y, J X, Y, J X)(k X-J X)\} \\
R(Y, J Y) X=\frac{1}{1+k^{2}}\{R(Y, J Y, X, J X)(k X-J X)\} \\
R(Y, J Y) Y=-H(Y)\{-k Y+J Y\} .
\end{gathered}
$$

Now define $X^{\prime}=a X+b Y$ with $a^{2}-b^{2}=1$, then using the above relations, we have

$$
R\left(X^{\prime}, J X^{\prime}\right) X^{\prime}=C_{1} X+C_{2} Y+C_{3} J X+C_{4} J Y
$$

where

$$
C_{3}=a^{3} H(X)\left(1+k^{2}\right)-\frac{a b^{2}}{\left(1+k^{2}\right)} C_{5}, \quad C_{4}=-b^{3} H(X)\left(1+k^{2}\right)+\frac{a^{2} b}{\left(1+k^{2}\right)} C_{5},
$$

and $C_{5}=R(X, J Y, Y, J X)+R(Y, J X, Y, J X)+R(Y, J X, X, J X)$. On the other hand, using (18), we have

$$
R\left(X^{*}, J X^{*}\right) X^{*}=-H\left(X^{*}\right)\{k a X+k b Y-a J X-b J Y\} .
$$


Comparing (19) and (20), we obtain

$$
a^{2} H(X)-\frac{b^{2}}{\left(1+k^{2}\right)} C_{5}=H\left(X^{*}\right), \quad-b^{2} H(X)+\frac{a^{2}}{\left(1+k^{2}\right)} C_{5}=H\left(X^{*}\right)
$$

from these equations, we obtain $C_{5}=\left(1+k^{2}\right) H(X)$ and consequently $H\left(X^{*}\right)=$ $\left(a^{2}-b^{2}\right) H(X)=H(X)$. Similarly, we can prove $H\left(Y^{*}\right)=H(Y)$. Thus $M$ is of constant holomorphic sectional curvature at $p$.

\section{References}

[1] N. Degirmenci and S. Karapazar, Spinors on Kaehler Norden manifolds, J. Nonlinear Math. Phys., 17, No. 1 (2010), 27-34.

[2] G. Ganchev, K. Gribachev and V. Mihova, Holomorphic hypersurfaces of Kaehler manifolds with Norden metric, Plovdiv Univ. Sci. Works Math., 23, No. 2 (1985), 221-236 (in Bulgarian).

[3] G. Ganchev and A. Borisov, Note on the almost complex manifolds with a Norden metric, Compt. Rend. Acad. Bulg. Sci., 39, No. 5 (1986), 31-34.

[4] G. Ganchev, V. Mihova and K. Gribachev, Almost contact manifolds with B-metric, Math. Balkanica (N.S.), 7 (1993), 261-276.

[5] A. Gray, Nearly Kaehler manifolds, J. Differential Geom., 4 (1970), 283-309.

[6] Rakesh Kumar, Rachna Rani and R. K. Nagaich, Constancy of $\phi$-holomorphic sectional curvature in indefinite Sasakian manifolds, Int. Elec. J. of Geom., 2 (2009), 34-40.

[7] M. Manev and K. Gribachev, Conformally invariant tensors on almost contact manifolds with B-metric, Serdica Math. J., 20 (1994), 133-147.

[8] R. K. Nagaich, Constancy of holopmorphic sectional curvature in indefinite almost hermition manifolds, Kodai. Math. J., 16 (1993), 327-331.

[9] A. P. Norden, On a class of four-dimensional A-spaces, Russian Math., 17, No. 4 (1960), 145-157.

[10] S. Tano, Constancy of holopmorphic sectional curvature in almost hermition manifolds, Kodai. Math. Sem. Rep., 25 (1973), 190-201. 